Versicherungspflicht für Elementarschäden

\section{Mehr Klimaschutz durch stete Kosten}

\author{
Nach dem Hurrikan Katrina streiten in den USA Versicherungen \\ darüber, ob die Schäden vom Sturm oder von der Flut verursacht \\ wurden. Sturmschäden sind gedeckt, Flutschäden nicht. Der \\ Streit zeigt, wie unvernünftig eine selektive Katastrophenversi- \\ cherung ist. Eine Pflichtversicherung für Hausbesitzer ist des- \\ wegen angezeigt. Von Reimund Schwarze und Gert G. Wagner
}

D ie Katastrophe von New Orleans verdeckt in den Schlagzeilen, dass auch Europa in diesem Sommer von zahlreichen „Elementarschäden“ betroffen war. Starkregen und Überschwemmungen haben erstmals wieder in Deutschland und Österreich Menschenleben gekostet sowie Millionenschäden verursacht.

Diese Unwetterepisode zeigt endgültig: Die Überschwemmungsgefahr, verbunden mit anderen Elementarrisiken wie Sturm oder Dürre, ist bei zunehmenden Wetterextremen auch in Europa ein allgemeines Lebensrisiko geworden. Trotzdem treffen Elementarschäden die Bürger immer wieder unvorbereitet und daher mit besonderer Härte. Angesichts der nicht endenden Kette von Ereignissen brauchen Europa und Deutschland endlich eine übergreifende Strategie der Vorsorge gegen Naturkatastrophen.

\section{Versicherungswirtschaft hat breite Schultern}

Eine Versicherungspflicht, die Gebäude gegen Elementarschäden aller Art absichern würde und Anreize zur planmäßigen Prävention setzt, sollte ein Eckstein einer solchen Strategie sein. Auch Katrina wird am Ende wieder zeigen: Die Versicherungswirtschaft kann große Schadenssummen schultern. Das Problem privater Lösungen ist deren selektive Deckung, unter der gerade die Armen einer Gesellschaft leiden. Da Überflutungen und Sturmschäden im Zuge des Klimawandels kein lokal begrenztes Problem sind, sind Bau- und Benutzungsverbote keine überzeugende Antwort.

Gerade die Deutschen lieben die Nähe zum Wasser. Mehr als 16 Prozent der flussnahen Flächen in Deutschland sind heute als Bauland genutzt, flussferne Gebiete sind nur zu knapp acht Prozent bebaut. Ein Trend, der sich fortsetzen wird. Grundsätzlich ist er auch nicht zu beanstanden, solange die Kosten des Wohnens mit Flussblick nicht von der Allgemeinheit getragen werden.

Wer flussnah oder im Alpenvorland leben will, sollte auch die damit verbundenen Hochwasser-Risken tragen. Versicherungen, die ständig Kosten verursachen, gewährleisten dies und sichern gleichzeitig ab. Im Nachgang der Ereignisse an der Oberelbe im Sommer 2002 wurden von den Finanzministern und der Versicherungswirtschaft still und durchaus konstruktiv die Möglichkeiten einer Versicherungspflicht gegen Elementarschäden diskutiert, die von der privaten Versicherungswirtschaft hätte realisiert werden sollen.

Das Vorhaben scheiterte allerdings, da es ohne staatliche Garantien nicht machbar schien. Angesichts der großen positiven Wirkungen einer Elementarschadenversicherung muss man eins nüchtern feststellen: Wenn die freiwillige privatwirtschaftliche Lösung nicht machbar ist, dann muss man auch angesichts der Erfolge im Ausland über staatliche Monopollösungen nachdenken. In der Schweiz zum Beispiel wird im Rahmen der kantonalen Monopolversicherungen eine intel- ligent verzahnte, technische und finanzielle Vorsorge gegen Naturrisiken betrieben. 60 Prozent der Vorsorgekosten bringen dort die Privaten auf, in Deutschland sind es weniger als fünf Prozent. In Frankreich werden von einer Pflichtversicherung Gebäudedeckungen gegen Naturkatastrophen für ca. 30 Euro Jahresprämie angeboten. Die "Consorcio“ in Spanien bietet ein weiteres Beispiel dafür, das offenbar auch EU-konform ist.

\section{Versicherungen kein Allheilmittel}

Auch Versicherungen können Naturereignisse nicht verhindern. Aber im Schadensfalle wird nicht nur eine planmäßige Entschädigung gezahlt, vielmehr zwingen uns klug gestaltete Versicherungen zu vernünftigerem Verhalten, da die Kosten von Risiken uns durch die Versicherungsprämien ständig vor Augen geführt werden.

Ähnlich wie der demografische Wandel nur zum Thema wurde, weil die Beiträge zu den Pflichtversicherungen für die Risiken Pflege und Rente massiv davon beeinflusst werden, würde durch eine Versicherungspflicht für Elementarschäden der Klimaschutz permanent in der öffentlichen Diskussion sein. Dadurch würden die betroffenen Bürger sich nicht nur individuell mehr Gedanken machen, wie sie durch präventive Maßnahmen an ihren Häusern die Kosten für die Versicherungsprämien senken könnten, sondern würden als Wähler und Wählerinnen auch wirksamere kollektive Maßnahmen für den Klimaschutz erzwingen. $\leftarrow$

I AUTOREN + KONTAKT

Dr. Reimund Schwarze ist Professor für Volkswirtschaftslehre an der Europa Universität Viadrina.

Dr. Gert G. Wagner ist Professor für Volkswirtschaftslehre an der TU Berlin. Beide kooperieren am Deutschen Institut für Wirtschaft (DIW).

DIW, Königin-Luise-Straße 5, 14195 Berlin. Tel. 030/89789-0, E-Mail: rschwarze@diw.de, gwagner@diw.de 
(c) 20I0 Authors; licensee IÖW and oekom verlag. This is an article distributed under the terms of the Creative Commons Attribution Non-Commercial No Derivates License (http://creativecommons.org/licenses/by-nc-nd/3.o/), which permits unrestricted use, distribution, and reproduction in any medium, provided the original work is properly cited. 